\title{
The Burden of Type 2 Diabetes and the Value of Achieving Near Normoglycemia from the Patient Perspective
}

Heather Gelhorn · Zaneta Balantac · Shraddha Shinde •

Vivian Thuyanh Thieu $\cdot$ Kristina S. Boye

Received: January 19, 2021 / Accepted: March 24, 2021 / Published online: May 27, 2021

(C) The Author(s) 2021

\section{ABSTRACT}

Introduction: Type 2 diabetes (T2D) is extremely burdensome to people with $\mathrm{T} 2 \mathrm{D}$ and associated with impaired health-related quality of life. This study explores the impact of T2D and potentially relevant outcomes for new therapies using a unique approach to in-depth qualitative interviews where people with T2D are asked to think about their future with T2D. Methods: A cross-sectional qualitative interview study among people with T2D from the USA and UK. Interviews explored their

Supplementary Information The online version contains supplementary material available at https:// doi.org/10.1007/s13300-021-01054-6.

H. Gelhorn $(\bowtie) \cdot$ Z. Balantac

Evidera Inc., 7101 Wisconsin Ave, Suite 1400, Bethesda, MD, USA

e-mail: Heather.Gelhorn@evidera.com

\section{Z. Balantac}

e-mail: zaneta.balantac@evidera.com

S. Shinde - V. T. Thieu - K. S. Boye

Eli Lilly and Company, Indianapolis, IN, USA

e-mail: shinde_shraddha@lilly.com

V. T. Thieu

e-mail: thieu_vivian_thuyanh@lilly.com

K. S. Boye

e-mail: boye_kristina_secnik@lilly.com treatment journey, perceptions of their future with T2D, and the value of achieving normoglycemia (explored through presentation of two vignettes with hypothetical medications that reduced hemoglobin A1c [HbA1c] levels $<7 \%$ and $<5.7 \%$ ).

Results: Patients with T2D ( $N=50$; US $n=25$; UK $n=25$ ) were $66.0 \%$ male, had a mean body mass index (BMI) of $30.8 \pm 6.3 \mathrm{~kg} / \mathrm{m}^{2}$, and had a mean of $13.0 \pm 10.0$ years since diagnosis. Current diabetes treatments included diet and exercise only $(8.0 \%)$, oral medications only $(62.0 \%)$, oral plus injections $(24.0 \%)$, and insulin only $(6.0 \%)$. Despite being treated, participants reported over 25 different unmet needs related to their T2D across a broad range of domains. The most common concerns were diet, diabetes-related complications, weight changes, and psychological and emotional issues. A large majority of participants indicated that achieving lower HbA1c values would change their life. When reflecting on the value of improved glycemic control, patients primarily anticipated physical improvements and improved psychological well-being. When presented with two hypothetical treatments, about $70 \%$ of participants preferred the $<5.7 \%$ treatment option over the $<7 \%$ HbA1c treatment option.

Conclusions: People with T2D have a high disease burden, a broad range of unmet needs, and extremely varied experiences and expectations on the impacts of T2D on their lives and 
future. Many patients indicated that achieving near normoglycemia would substantially change their lives primarily in terms of their physical and emotional health.

Keywords: Diabetes; Disease burden; HbA1c; Normoglycemia; Type 2 diabetes; Unmet needs

\section{Key Summary Points}

Why carry out this study?

As novel therapies are developed with potential for better outcomes, including the possibility of achieving near normoglycemia, it is important to understand how people with type 2 diabetes (T2D) currently view the burden of the disease and the benefits and risks associated with new treatment options

Most prior qualitative studies have explored disease burden based on the past and current experiences of individuals with T2D. The current study explores the impact of T2D and relevant outcomes for new therapies using a unique approach in which participants with T2D are asked to think about their future and verbalize what is most concerning and important to them. Through this approach, it was hoped that people with T2D could more easily prioritize and communicate the diabetes-related outcomes that are most meaningful to them

A key area of investigation in the current study was to explore how people with T2D might perceive achieving near normoglycemia, understand their perceptions of potential risk-benefit tradeoffs (e.g., HbA1c, weight changes, side effects), and explore the specific benefits beyond HbA1c that were anticipated from the perspective of individuals with T2D

\section{What was learned from the study?}

The results of this study showed that a treatment that achieves near normoglycemia without increasing the risk of hypoglycemia would be a paradigm shift for many individuals with T2D and a highly valued option with many anticipated benefits. In addition to lowered HbA1c values, reductions in psychological and emotional distress and physical benefits would be the primary areas in which people with T2D would expect to experience meaningful improvements

There is still a substantial burden of disease for T2D that is not addressed by current treatments. New treatment options would be welcomed by people with T2D, though they would be evaluated by individual patients (and likely their physicians) in light of the associated risks, including side effects and, for some, the substantial weight loss

\section{DIGITAL FEATURES}

This article is published with digital features, including a summary slide, to facilitate understanding of the article. To view digital features for this article go to https://doi.org/10.6084/ m9.figshare.14249138.

\section{INTRODUCTION}

Type 2 diabetes (T2D) is a serious condition, and the prevalence has risen dramatically over the past 3 decades in both developed and developing countries [1-3]. This number is projected to rise to 578 million, or $10.2 \%$ of the global population, by 2030 and to 700 million (10.9\%) by 2045 [4]. Worsened by both an increasing elderly population and rising obesity rates [5-10], T2D now accounts for $90 \%$ of all diabetes cases $[6,11]$ and is currently the most common metabolic disorder worldwide. 
People with T2D find their condition to be extremely burdensome, and it is often associated with impaired health-related quality of life (HRQoL) [12, 13]. Diabetes, especially with poor glycemic control, is associated with higher risks of serious complications and comorbidities, including coronary artery disease, stroke, peripheral vascular disease, retinopathy, nephropathy, and neuropathy $[3,14,15]$.

Currently, there is a dearth of published qualitative research on the unmet needs of people with T2D. These types of studies can provide a patient-centered approach to gathering rich information on the nature, extent, and perceived importance of the continued challenges that people with T2D face. Poor psychological well-being, physical health status, lifestyle behavior, and social environments are several factors that have been previously found to be associated with diabetes [14, 16-18]. However, most prior qualitative studies have explored disease burden based on the past and current experiences of individuals with T2D. These retrospective and contemporaneous approaches yield a wealth of data but may make it more difficult to understand which aspects of their condition are the most important to patients and thus critical outcomes for the evaluation of novel therapies. The current study explores the impact of T2D and potentially relevant outcomes for new therapies using a unique approach in which participants with T2D are asked to think about their future with T2D and verbalize what is most concerning and important to them.

Achieving glycemic control is a crucial step to diabetes management and may reduce the risk of future complications. Current guidelines from the American Diabetes Association (ADA) recommend that people with T2D achieve a goal of $<7 \%(53 \mathrm{mmol} / \mathrm{mol})$ hemoglobin A1c (HbA1c) [19]. Treatment in combination with lifestyle modifications is often recommended to achieve this goal [19], and advancements in the last 10 years have led to new approaches to therapy and made this glycemic goal easier to achieve [20]. More recently, research has shown that movement from above target $\mathrm{HbA1c}$ to normoglycemia (HbA1c $<5.7 \%$ ), a blood glucose level similar to people without diabetes, may also be achieved therapeutically [21]. To date, achieving near normoglycemia has rarely been a focus, or even considered, by people with T2D or their treatment providers.

Little is known about what achieving normoglycemia would mean to people with T2D. Given the distinct possibility that this may now be achievable for at least some individuals with $\mathrm{T} 2 \mathrm{D}$, this study examined the perceived value of achieving near normoglycemia (HbA1c $<5.7 \%$ ) among people with T2D. In the current study, participants with T2D were asked to imagine the future and anticipate how their lives could be different should they achieve near normoglycemia. Through this approach, it was hoped that people with T2D could more easily prioritize and communicate the diabetes-related outcomes that are most meaningful to them.

\section{METHODS}

\section{Sample}

This was a cross-sectional qualitative study involving interviews with adult participants with self-reported T2D. Participants were recruited through newspaper and online advertisements in the USA and UK. Key inclusion criteria included age $\geq 18$ years at the time of screening and self-reported diagnosis of T2D as diagnosed by a medical professional. Key exclusion criteria included diagnosis of type 1 diabetes mellitus or gestational diabetes, cognitive, physical, or psychological impairment, or insufficient knowledge of English that would interfere with their ability to provide consent or complete the interview.

\section{Measures and Procedures}

Interviews were conducted one on one either in person or by telephone/web conference using a standardized interview guide. The interview guide was developed based on key questions of interest and discussions with all members of the study team (see Supplemental material for example interview questions). Participants were asked about their T2D journey and treatment 


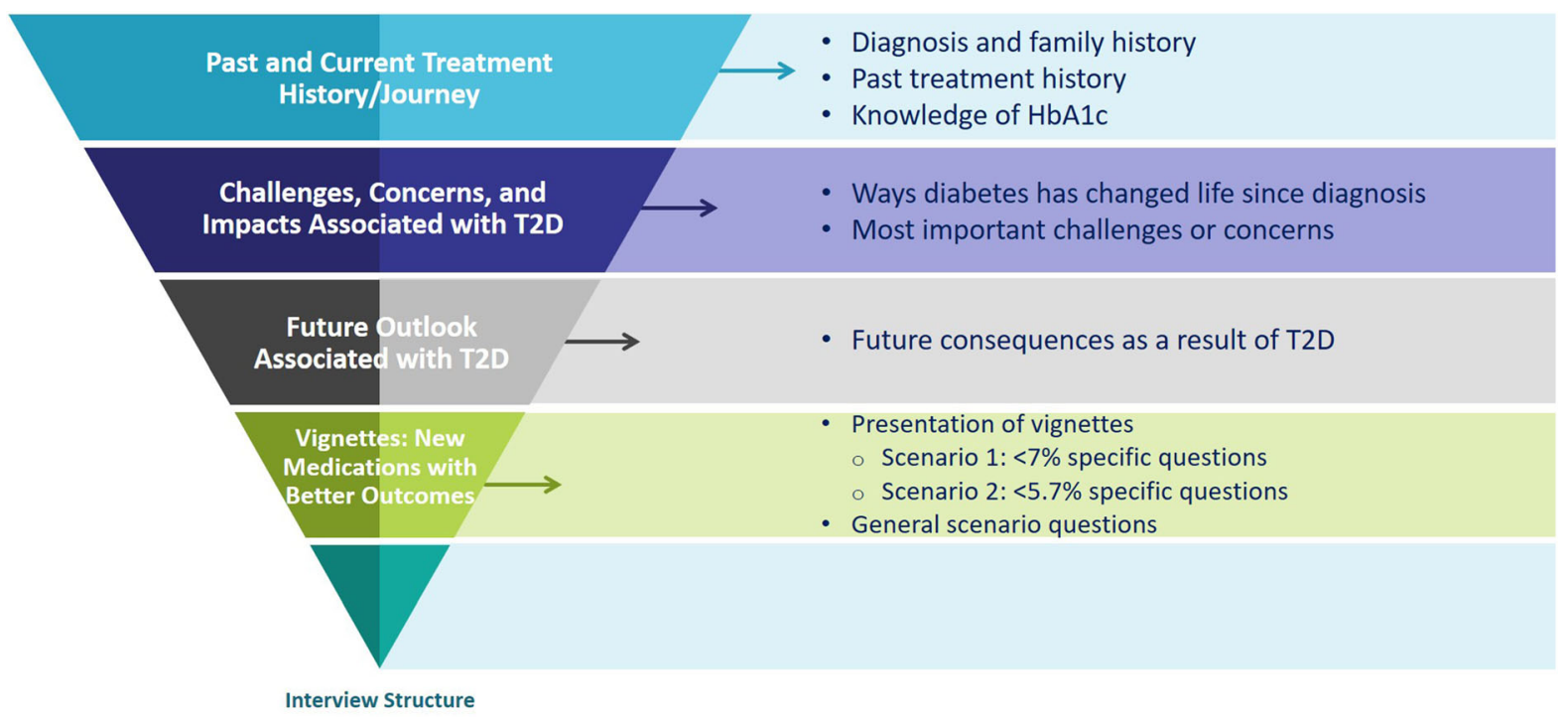

Fig. 1 Interview flow

history, the challenges and changes they experienced as a result of their diagnosis, the ways in which diabetes has changed their lives, and perceptions of their future with T2D (Fig. 1). The interviews also included two vignettes (see Supplementary Figures S1a and S1b) that were used to understand the value of achieving normoglycemia (HbA1c target $<5.7 \%$ ) from the perspective of people with T2D. These vignettes, developed based on results from a recent phase 2 trial [21], presented two hypothetical treatments with similar administration profiles; both treatments are non-insulin auto-injections to be taken once a week using a single-dose use pen. After 6 weeks of treatment, one treatment would lower HbA1c levels to about 7\% (Figure S1a) with an average weight loss of $6 \mathrm{lbs}$ and a $35 \%$ chance of gastrointestinal (GI) side effects. In contrast, the second treatment would lower HbA1c levels to $5.7 \%$ or lower with an average weight loss of $25 \mathrm{lbs}$ and a $60 \%$ chance of GI side effects.

Participants were asked to complete sociodemographic and clinical questions at the end of the interview through a web-based application. All participants provided informed consent prior to the interviews and were remunerated for their participation. All procedures performed were in accordance with the ethical standards of the institutional and/or national research committee and with the 1964 Helsinki Declaration and its later amendments or comparable ethical standards. The study protocol and all study procedures were approved by an institutional review board prior to initiating the study (Ethical and Independent Review Services, protocol \#20010-01, v1.0 date 6 January 2020, approved 11 February 2020).

\section{Analyses}

All interviews were digitally recorded and transcribed. Qualitative data were analyzed using ATLAS.ti version $8.0+[22]$, a data analysis software program commonly used to organize and categorize text in interview transcripts. Using a methodology described by Willis (2015) and Hsieh \& Shannon (2005), the de-identified data were examined to evaluate the underlying structure of the qualitative data [23, 24]. A coding framework was developed based on the structure of the interview guide. The first several transcripts were independently coded, and the coding was compared for consistency. An iterative, comparative coding approach was used as coding continued between consecutive transcripts and new codes emerged [25]. Any new codes identified were subsequently added to the coding framework. Participant quotes were then grouped and summarized by thematic code, and 
Table 1 Self-reported sociodemographic and clinical characteristics

\begin{tabular}{|c|c|c|c|}
\hline Sociodemographic and clinical characteristics & $\begin{array}{l}\text { Total } \\
N=\mathbf{5 0}\end{array}$ & $\begin{array}{l}\text { US } \\
N=25\end{array}$ & $\begin{array}{l}\text { UK } \\
N=25\end{array}$ \\
\hline \multicolumn{4}{|l|}{ Age (years) } \\
\hline Mean (SD) & $63.7(11.3)$ & $68.9(8.5)$ & $58.5(11.5)$ \\
\hline \multicolumn{4}{|l|}{ Gender, n (\%) } \\
\hline Male & $33(66 \%)$ & $17(68 \%)$ & $16(64 \%)$ \\
\hline \multicolumn{4}{|l|}{ Race $^{a} \mathrm{n}(\%)$} \\
\hline Asian & $7(14 \%)$ & $2(8 \%)$ & $5(20 \%)$ \\
\hline Black/African/Caribbean & $5(10 \%)$ & $1(4 \%)$ & $4(16 \%)$ \\
\hline White & $35(70 \%)$ & $21(84 \%)$ & $14(56 \%)$ \\
\hline Other $^{\mathrm{b}, \mathrm{c}}$ & $3(6 \%)$ & $1(4 \%)$ & $2(8 \%)$ \\
\hline \multicolumn{4}{|l|}{ Employment status, n (\%) } \\
\hline Employed, full-time & $12(24 \%)$ & $4(16 \%)$ & $8(32 \%)$ \\
\hline Employed, part-time & $5(10 \%)$ & $2(8 \%)$ & $3(12 \%)$ \\
\hline Retired & $21(42 \%)$ & $15(60 \%)$ & $6(24 \%)$ \\
\hline Other $^{\mathrm{d}}$ & $12(24 \%)$ & $4(16 \%)$ & $8(32 \%)$ \\
\hline \multicolumn{4}{|l|}{ Body mass index (BMI; $\left.\mathrm{kg} / \mathrm{m}^{2}\right)$} \\
\hline Mean (SD) & $30.8(6.3)$ & $31.1(6.5)$ & $30.5(6.2)$ \\
\hline \multicolumn{4}{|l|}{ Time since diagnosis (years) } \\
\hline Mean (SD) & $13.0(10.0)$ & $16.2(11.9)$ & $9.9(6.6)$ \\
\hline \multicolumn{4}{|l|}{ Most recent laboratory (HbAlc) level, n (\%) } \\
\hline Below $6.5 \%(48 \mathrm{mmol} / \mathrm{mol})$ & $13(26 \%)$ & $5(20 \%)$ & $8(32 \%)$ \\
\hline Between $6.5 \%$ and $7 \%(48 \mathrm{mmol} / \mathrm{mol}$ and $53 \mathrm{mmol} / \mathrm{mol})$ & $9(18 \%)$ & $6(24 \%)$ & $3(12 \%)$ \\
\hline Between $7.1 \%$ and $8 \%(54 \mathrm{mmol} / \mathrm{mol}$ and $64 \mathrm{mmol} / \mathrm{mol})$ & $12(24 \%)$ & $10(40 \%)$ & $2(8 \%)$ \\
\hline Between $8.1 \%$ and $9 \%(65 \mathrm{mmol} / \mathrm{mol}$ and $75 \mathrm{mmol} / \mathrm{mol})$ & $3(6 \%)$ & $2(8 \%)$ & $1(4 \%)$ \\
\hline Higher than $9 \%(75 \mathrm{mmol} / \mathrm{mol})$ & $5(10 \%)$ & $2(8 \%)$ & $3(12 \%)$ \\
\hline Do not know & $8(16 \%)$ & $0(0 \%)$ & $8(32 \%)$ \\
\hline \multicolumn{4}{|c|}{ Type of treatment currently receiving for diabetes by combination, $\mathrm{n}(\%)$} \\
\hline Diet and exercise only & $4(8.0 \%)$ & $3(12.0 \%)$ & $1(4.0 \%)$ \\
\hline Oral/tablets only ${ }^{\mathrm{e}}$ & $31(62.0 \%)$ & $9(36.0 \%)$ & $22(88.0 \%)$ \\
\hline Insulin only ${ }^{\mathrm{e}}$ & $3(6.0 \%)$ & $3(12.0 \%)$ & $0(0.0 \%)$ \\
\hline Oral + insulin and/or non-insulin ${ }^{\mathrm{c}}$ & $12(24.0 \%)$ & $10(40.0 \%)$ & $2(8.0 \%)$ \\
\hline \multicolumn{4}{|l|}{ Description of overall health, $\mathrm{n}(\%)$} \\
\hline Excellent & $2(4 \%)$ & $0(0 \%)$ & $2(8 \%)$ \\
\hline Very good & $7(14 \%)$ & $7(28 \%)$ & $0(0 \%)$ \\
\hline Good & $27(54 \%)$ & $11(44 \%)$ & $16(64 \%)$ \\
\hline Fair & $13(26 \%)$ & $7(28 \%)$ & $6(24 \%)$ \\
\hline Poor & $1(2 \%)$ & $0(0 \%)$ & $1(4 \%)$ \\
\hline
\end{tabular}

${ }^{a}$ Not mutually exclusive

b Other race (US only) includes: 'Mexican' $(n=1)$

c Other race (UK only) includes: 'Greek Cypriot' $(n=1)$, 'White and Black Caribbean' $(n=1)$

d Other employment includes: 'disabled' $(n=4)$, 'homemaker/housewife' $(n=2)$, 'student' $(n=1)$, 'unemployed' $(n=1)$, 'carer' $(n=1)$, 'unpaid carer' $(n=1)$, 'COVID-19' $(n=1)$, and 'retired/self-employed' $(n=1)$

c Includes participants who selected and did not select "diet and exercise"

coding outputs were generated based on each utilized code.
Descriptive statistics were used to characterize the sample. Means, standard deviations (SDs), and ranges were presented for continuous 


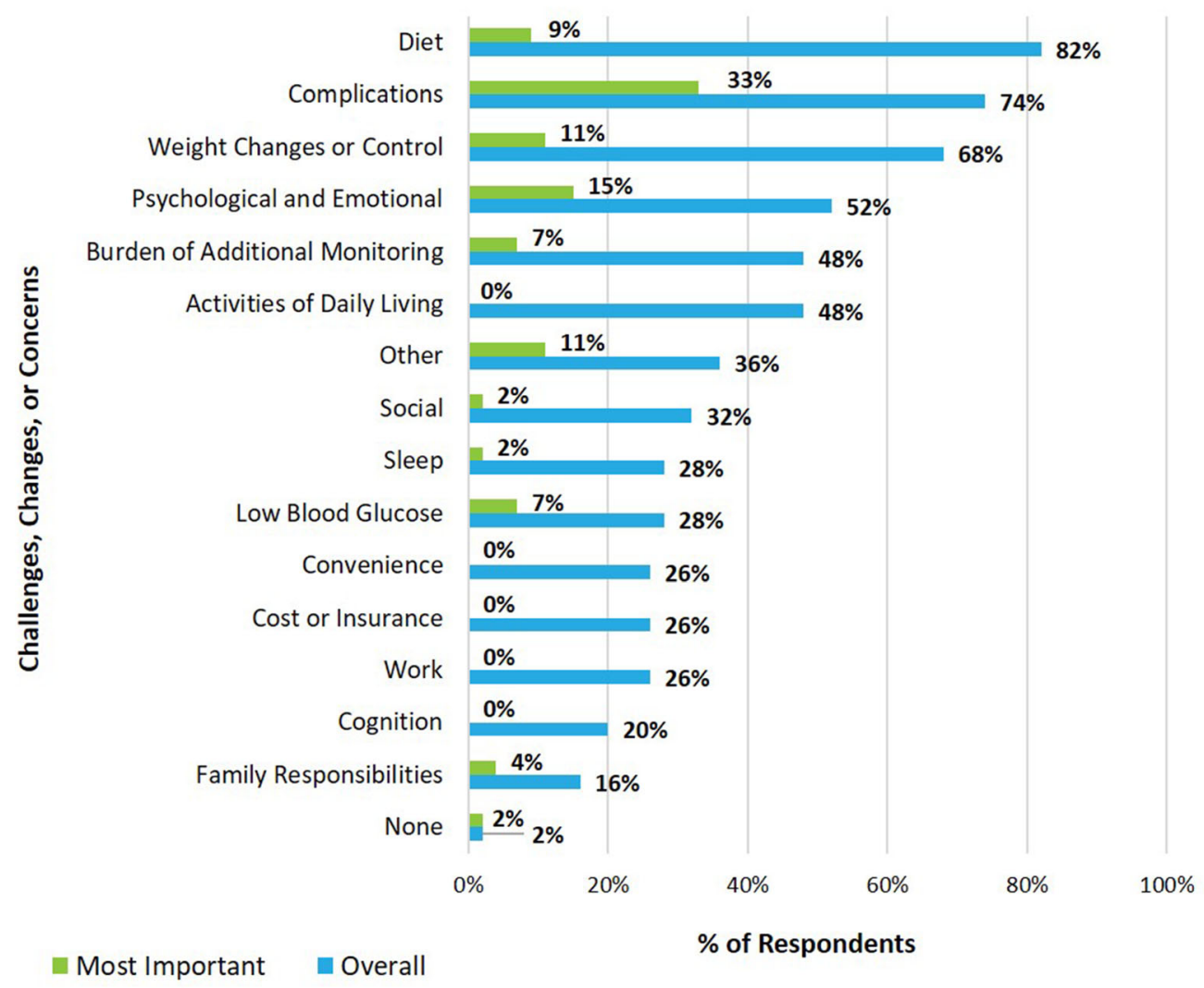

Fig. 2 Challenges, changes, or concerns due to T2D. a Other includes: challenges of tiredness, fatigue, or lack of energy $(n=5)$, concerns of taking too many tablets $(n=5)$, fear of taking injections $(n=2)$, risk of family members getting diabetes $(n=2)$, concerns of keeping HbAlc low $(n=2)$, frequency of administration $(n=1)$, fear of worsening immune system $(\mathrm{n}=1)$, concerns of increased

variables, and frequencies and percentages were presented for categorical variables. Analyses were conducted using SAS version 9.4 (SAS Institute, Cary, NC).

\section{RESULTS}

A total of 50 people with T2D completed interviews, including 25 participants from the USA and 25 from the UK. Self-reported sociodemographic characteristics are presented in Table 1 . The mean age of the participants was 63.7 years (SD: 11.3 ; range: $24-85$ ), and $66 \%$ $(n=33)$ were male. The majority of the participants in each country identified as White (US: vulnerability $(n=1)$, fear of worsening health $(n=1)$, and fear of treatment side effects $(n=1)$. b Percentages are based on the number of respondents for each question. $\mathrm{N}=50$ responded to overall challenges, changes, or concerns due to T2D. N=46 responded to the most important challenge, change, or concern

$n=21,84 \%$; UK: $n=14,56 \%)$, with some representation of minorities in each country. In the US, most of the participants were retired $(n=15,60 \%)$, while four reported full-time employment $(16 \%)$. In the UK, close to one third of the participants were employed fulltime $(n=8,32 \%)$, and six were retired $(24 \%)$. There was a good distribution of participants across the different education levels in each country.

Although almost all participants (92\%) were currently being treated for T2D therapeutically, over 25 unmet needs related to their T2D were reported across a broad range of domains. Participants described how T2D affects their life, including challenges, changes, and concerns 


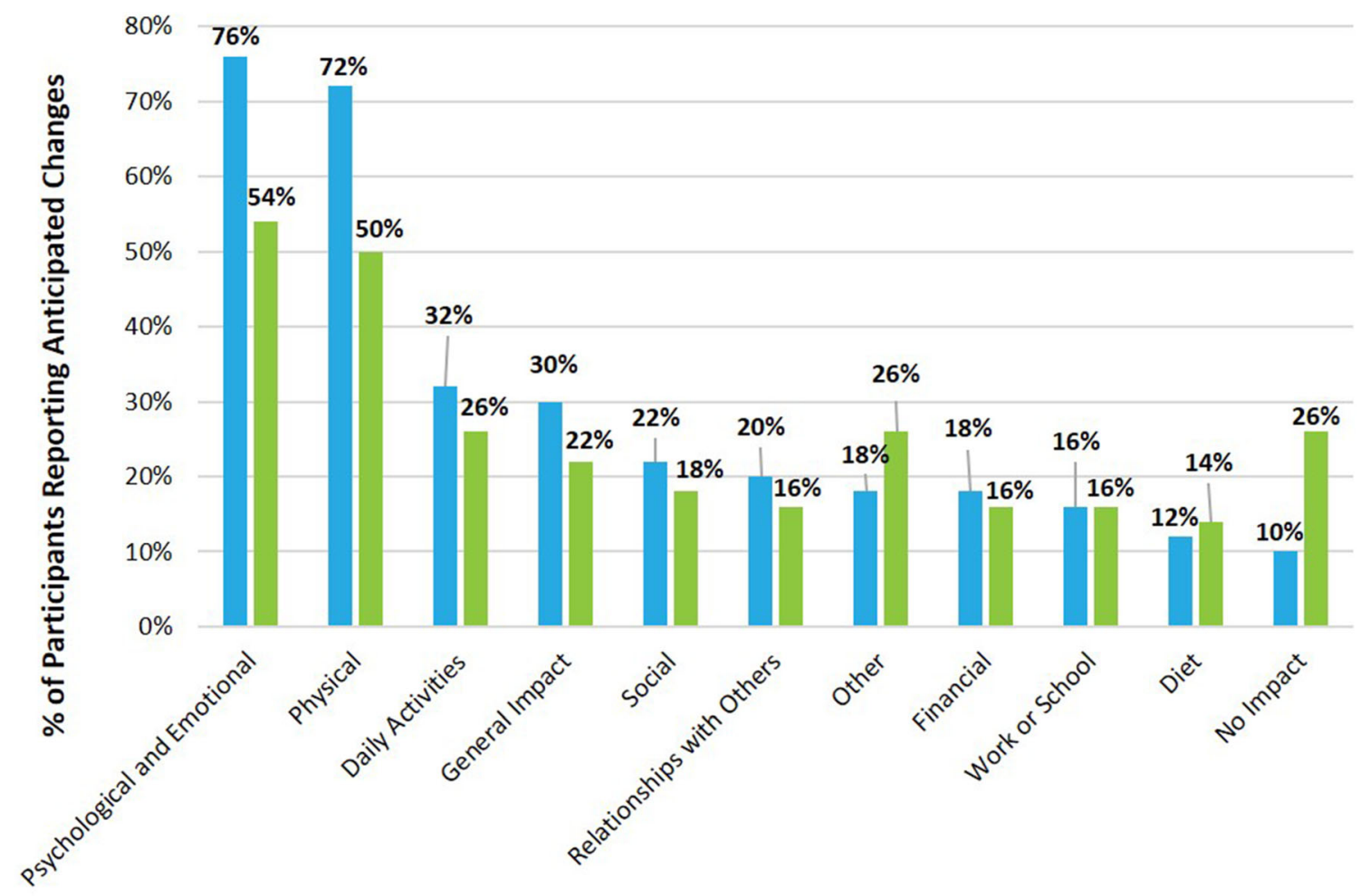

Anticipated Changes

Yes to $<5.7 \%$ treatment $\square$ Yes to $<7 \%$ treatment

Fig. 3 Anticipated changes in participants' lives with lower HbAlc. a $\mathrm{N}=50$ responded to anticipated changes (e.g., psychological and emotional). b Other includes: better convenience (e.g., fewer doctor visits, less time required for treatment administration; $<5.7 \% \mathrm{n}=7,<7 \%$ $n=7$ ), reduced treatment or frequency of treatment (e.g.,

they have experienced because of T2D. The most common thematic responses described by participants were challenges, changes, or concerns related to diet $(82 \%)$, health complications related to T2D (74\%), and weight changes or control (68\%) (Fig. 2). Close to $50 \%$ of the participants also brought up concepts in the domains of psychological and emotional burden (52\%), activities of daily living (48\%), and burden of additional monitoring (48\%). When asked which of these was most important to them, participants most commonly selected complications (33\%).

As part of the interview, participants were presented with two scenarios describing two once-a-week fewer pills, once a week; $<7 \% \mathrm{n}=5$ ), less monitoring $(<5.7 \% \mathrm{n}=2)$, increased complacency or recklessness $(<5.7 \% \mathrm{n}=1,<7 \% \mathrm{n}=3)$, increased quality of life $(<5.7 \%$ $n=1)$, reduced likelihood of going on insulin $(<7 \% n=1)$, better cognition $(<7 \% \mathrm{n}=1)$, and reduced doctor concerns $(<7 \% \mathrm{n}=1)$

injectable medications. In the first medication profile scenario, participants would achieve HbA1c levels of about $7 \%$ and average weight loss was $6 \mathrm{lbs}$, and there was a 35\% chance of mild-to-moderate GI side effects at the beginning of therapy. In the second medication profile scenario, participants would achieve HbA1c levels of $5.7 \%$ or lower and average weight loss was $25 \mathrm{lbs}$, and there was a $60 \%$ chance of mild-to-moderate GI side effects at the beginning of therapy. Participants were asked to provide feedback on whether achieving the HbA1c goal under the conditions stated in each scenario would change their perceptions of their future. Additionally, participants were asked whether the HbA1c levels would change 

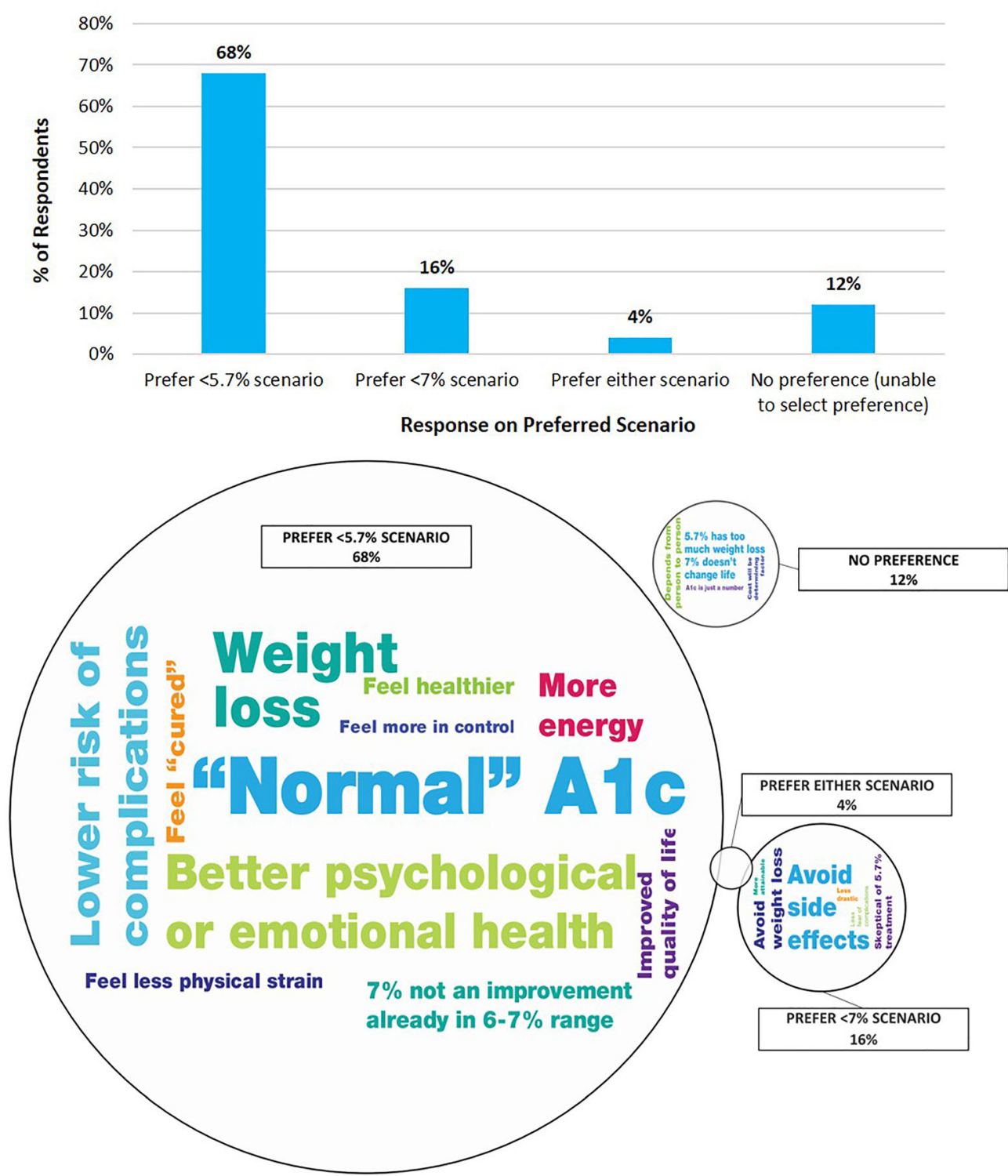

Fig. 4 Participants' preferences for medication scenarios and rationale

their lives and, if so, how. For those participants who had already achieved the HbA1c levels mentioned in the scenarios, the interview questions were modified to ask how this had impacted their lives in the past tense. Most participants $(83 \%)$ mentioned that both hypothetical treatments would change their perceptions of their future with diabetes (Fig. 3). More participants were optimistic about the future with the $<5.7 \%$ treatment scenario $(80 \%)$ compared to the $<7 \%$ treatment scenario $(66 \%)$.

A large majority of participants indicated that achieving lower HbA1c values would change their life and their perception of their future with diabetes. Irrespective of the treatment scenario they discussed, the most commonly anticipated impacts on the participants' lives and future perceptions were psychological and emotional in nature (86\%), including an increased sense of security, better quality of life, 


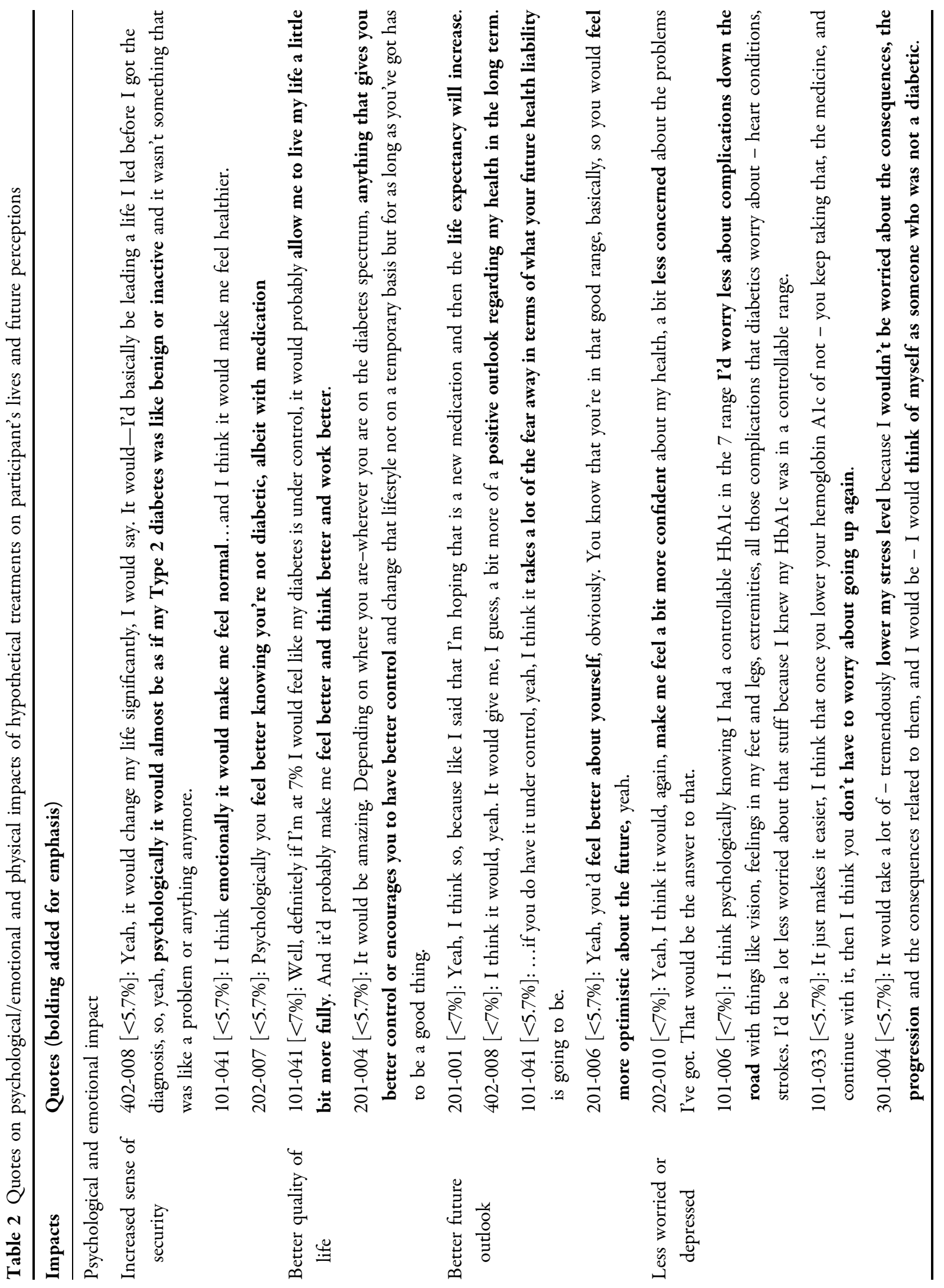




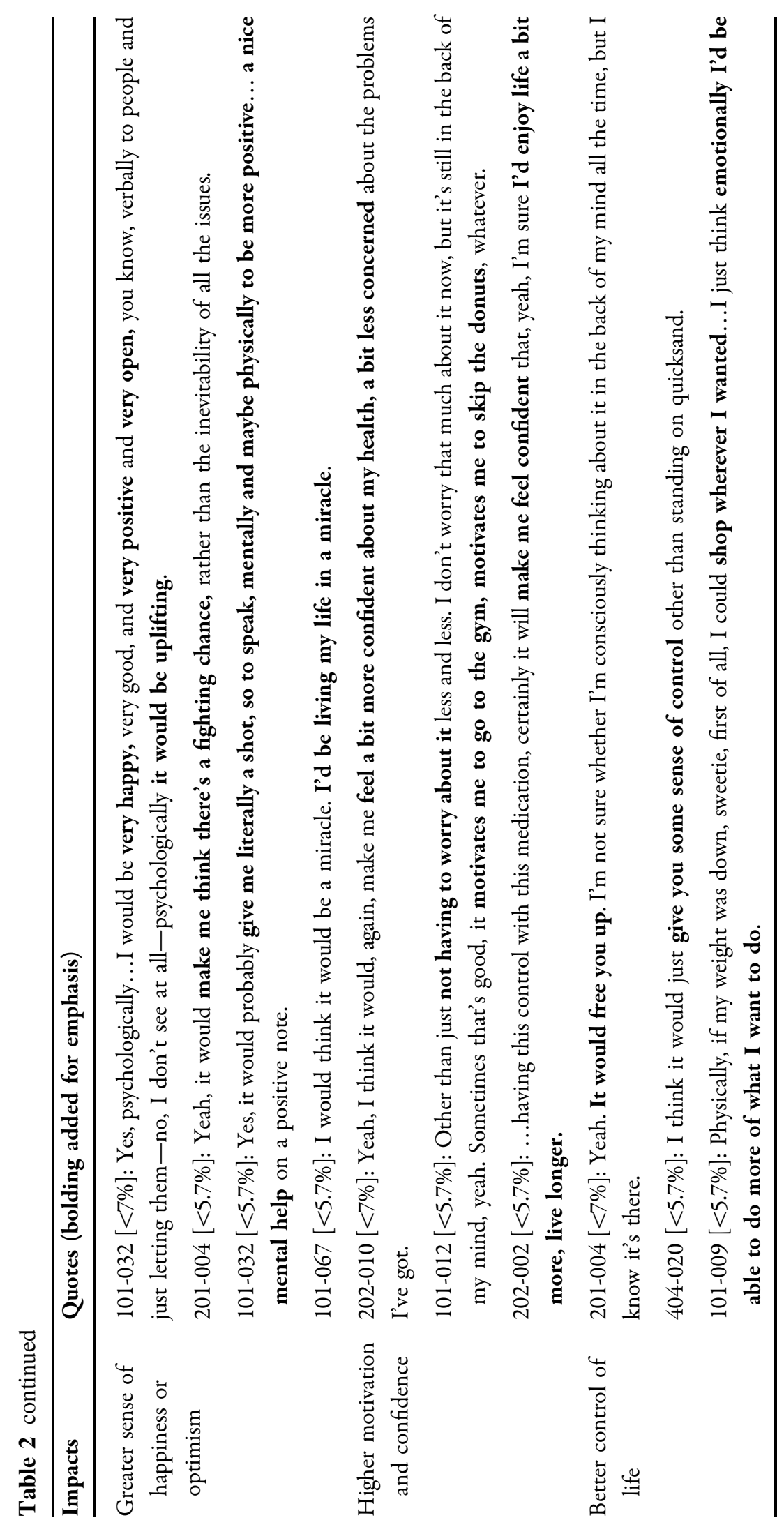




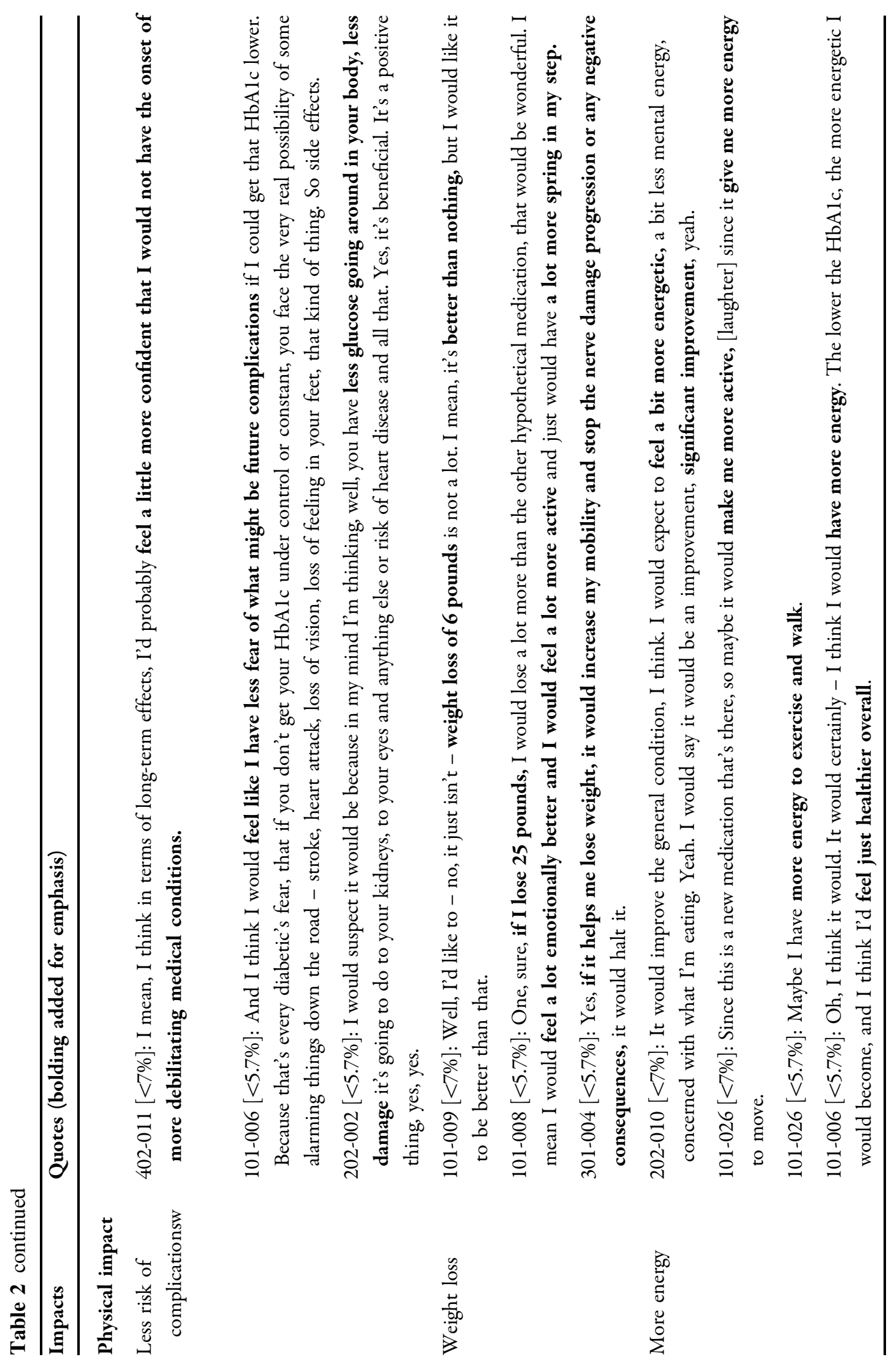




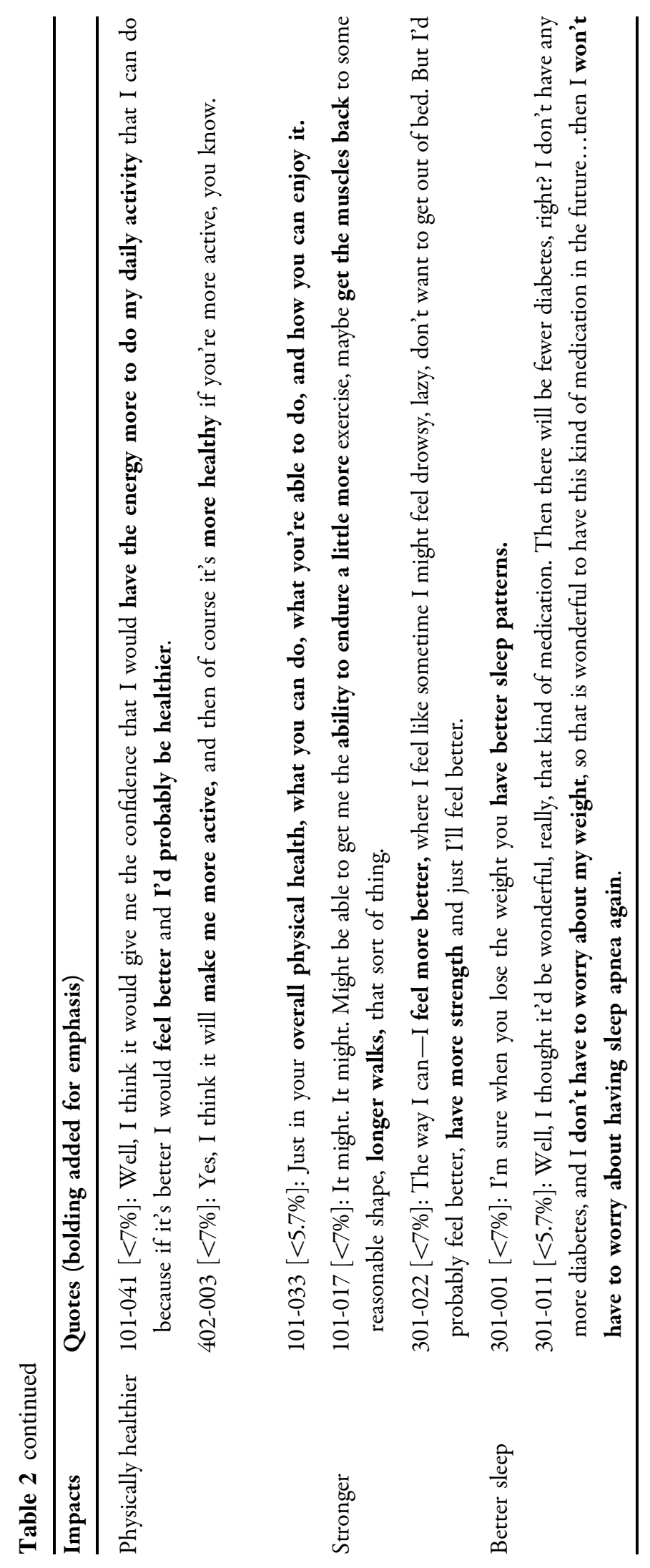


better outlook for their future, feeling less worried or depressed, feeling a greater sense of happiness or optimism, having higher motivation and confidence, and anticipating better control over their lives (Table 2). Many participants also mentioned physical improvements (82\%), including a lower risk of experiencing complications, less long-term damage to their bodies, weight loss, having more energy, bodies that were more physically healthy, having a stronger body, and better sleep. Sample quotes are provided below.

101-006 [<5.7\%]: And I think I would feel like I have less fear of what might be future complications if I could get that HbA1c lower. Because that's every diabetic's fear, that if you don't get your HbA1c under control or constant, you face the very real possibility of some alarming things down the road - stroke, heart attack, loss of vision, loss of feeling in your feet, that kind of thing.

202-002 [<5.7\%]: The benefits being it would help my other conditions I've already got through diabetes, one being the eyesight, the retinopathy. I know the 5.7 will help me. Not so much the 7\%. Having a low blood glucose level will definitely be the one I would ask for, a medication that will do that, if Scenario No. 2 would do that for me.

202-011 [<5.7\%]: I just think, well, I think psychologically it's going to have an effect because you know you're within that range. If it's now lower than 5.7, you know you're going to be less at risk of heart attacks, strokes, etc. If it's higher, your HbA1c is a high level, you know you're more pushing toward that risk category. So I just think it's, you know, the higher your HbA1c level is, the more stress and worry it is going to cause you.

301-004 [<5.7\%]: I would be much more - my stress level would be much higher at 7\%. I would be constantly worried about my blood sugar. At 7\% I'd be thinking about it every single day, every time I'd look at a needle, it would be going through my mind. Because anything above $7 \%$ is really bad, so it's like I'm on a ledge, you know. If it goes above 7\%, I fall, you understand? To me, 7\% is life- threatening. And I would worry about it, like I say, every single day.

Participants were also asked which of the two medication scenarios they preferred. The majority of participants preferred the $<5.7 \%$ scenario $(n=34 ; 68 \%)$, fewer preferred $<7 \%$ $(n=8 ; 16 \%)$, some could not select a preference $(n=6 ; 12 \%)$, and two (4\%) preferred either scenario (Fig. 4, top panel). The most common reasons mentioned by participants preferring the $<5.7 \%$ scenario were that they liked that the HbA1c would be significantly lowered and back to "normal" levels $(n=18)$. Many noted that the weight loss is significant $(n=13)$ and/ or that the treatment would reduce the risk of complications and long-term damage $(n=10)$ (Fig. 4, bottom panel). Participants who preferred the $<7 \%$ treatment scenario felt the risk of side effects in the $<5.7 \%$ scenario was too high $(n=7)$. Several participants also mentioned that they did not want to lose too much weight $(n=4 ; 8 \%)$; two of these participants had BMIs within the normal range $(18-25 \mathrm{~kg} /$ $\mathrm{m}^{2}$ ), while the other two had BMIs of 27 and 31, respectively. Among the participants who did not have a specific preference for either treatment, they felt that $7 \%$ would not have an impact on their life but also felt that $5.7 \%$ involved too much weight loss.

\section{DISCUSSION}

As novel therapies are developed with potential for better outcomes, including the possibility of achieving near normoglycemia [21], it is important to understand how people with T2D view the benefits and risks associated with new treatment options. The results of the current study and prior studies suggest that participants have extremely varied experiences and perspectives in terms of their diagnostic and treatment histories and their perceptions of the impact of T2D on their lives [26-29]. However, to date, there has been very little research on the perceptions and expectations of people with diabetes about their future with the condition. The aim of the current study was to gather these perspectives directly from individuals with $\mathrm{T} 2 \mathrm{D}$ 
through interviews that focused on how people with diabetes view their future. It was anticipated that this approach might help to highlight the outcomes and concerns that are most important to them. These findings may help identify currently unmet needs of people with T2D.

Despite the broad range of options, participants on currently available treatments for T2D continue to report a high disease burden and many unmet needs. A recent study suggests that both emotional burden and regimen-related distress are very high, particularly among people with T2D [30]. In the current study, the most common challenges and concerns described by participants were related to diet, complications, and weight changes or control, which was expected. However, more than half of the participants also highlighted psychological and emotional issues as a significant challenge, with this domain ranking second after complications in terms of what was most important to them. Consistent with this finding, it has previously been shown that T2D individuals with higher HbA1c levels have increased psychological and emotional distress [31-33]. In addition, prior studies have also found that individuals with T2D who show improvements in clinical measures through diabetes treatment also report improvements in psychological health [34-36].

A key area of investigation in the current study was to explore how people with T2D might perceive achieving near normoglycemia, understand their perceptions of potential risk-benefit trade-offs (e.g., HbA1c, weight changes, side effects), and explore the specific benefits beyond HbA1c that were anticipated from the patient perspective. When presented with two hypothetical treatments, the majority of participants (about 70\%) preferred the $<5.7 \%$ HbA1c treatment option over the $<7 \%$ treatment option. Many of the participants noted that they would be "back to normal" in terms of HbA1c, and the weight loss was often perceived as a significant benefit. Participants anticipated a reduced risk of complications and long-term physical damage to their bodies, which was often associated with expected decreases in psychological or emotional distress.
Not all participants preferred the treatment that achieved near normoglycemia. Almost universally, participants who did not prefer the $<5.7 \%$ treatment option cited the risk of side effects as too high to justify the reduction in HbA1c. In addition, some participants, half of whom had BMI within the normal range $\left(18.5-25 \mathrm{~kg} / \mathrm{m}^{2}\right)$, also felt that the weight loss associated with this treatment option was excessive (Fig. 4, lower panel). These results suggest that achieving normoglycemia would be highly valued by a majority of participants with T2D, and often preferred, despite a much larger risk of treatment side effects as compared with alternative options that offered more modest reductions in HbA1c. Regardless of the preferred treatment option, participants most frequently mentioned that they anticipated changes to their lives that were positive including 80\% anticipating benefits that were psychological, emotional, and/or physical. The anticipated benefits that were described by participants in the areas of psychological or emotional functioning suggest that future studies of treatments with the potential to achieve near normoglycemia might be best poised to identify relevant benefits by including measures that are designed to capture concepts such as levels of security, worry, depression, optimism, motivation, confidence, and control.

\section{Limitations}

The results of this study should be interpreted with consideration of the following limitations. The population recruited for this study was a convenience sample; participants were recruited through newspaper and online advertisements, and participants were older. Fewer participants than planned in the protocol were interviewed in-person due to COVID-19 restrictions; however, during the analyses no important differences were noted between those who completed telephone interviews versus in-person interviews. All participants in the study were included based on a self-reported diagnosis of their T2D. All participants were deemed sufficiently knowledgeable of T2D during the interviews to provide confidence in 
this diagnosis; however, no confirmation of diagnosis from a clinician or medication prescription was required. Lastly, the study design, which involved interviews to explore the impact of T2D and potentially relevant outcomes for new therapies, may have resulted in some selection bias. It is unknown to what extent those who volunteered to participate in the interviews represent the population of $\mathrm{T} 2 \mathrm{D}$ more generally.

\section{CONCLUSIONS}

A treatment that achieves near normoglycemia without increasing the risk of hypoglycemia would be a paradigm shift for many individuals with T2D and a highly valued option with many anticipated benefits. In addition to lowered $\mathrm{HbA} 1 \mathrm{c}$ values, reductions in psychological and emotional distress and physical benefits would be the primary areas in which people with T2D would expect to experience meaningful improvements. These treatment options would be welcomed by people with T2D, though they would be evaluated by individual patients (and likely their physicians) in light of the associated risks, including side effects and, for some, the substantial weight loss.

\section{ACKNOWLEDGEMENTS}

The authors would like to thank Cristina Abel, Fanyang Zeng, Ella Brookes, and Myrto Trapali for their assistance in executing the study. The authors would like to thank Fritz Hamme, Kawthar Nakayima, Amara Tiebout, Dawn Ri'chard, and Emily Sargent from Evidera for providing editing and production support throughout the study. The authors would also like to thank Amara Tiebout from Evidera for providing editing and production support during the writing of this article. All editing and production support services were funded by Eli Lilly and Company. Lastly, the authors would like to thank the participants of the study.
Funding. The study was funded by Eli Lilly and Company. Eli Lilly and Company also funded this journal's Rapid Service Fee.

Authorship. All named authors meet the International Committee of Medical Journal Editors (ICMJE) criteria for authorship for this article, take responsibility for the integrity of the work as a whole, and have given their approval for this version to be published.

Authorship Contributions. All authors participated in the design of the study. Heather Gelhorn and Zaneta Balantac participated in the execution of the study. All authors participated in the interpretation of data, review, and approval of the manuscript.

Disclosures. Heather Gelhorn and Zaneta Balantac are employees of Evidera, an independent research organization that received support from Eli Lilly to conduct this work. Kristina S. Boye, Vivian Thuyanh Thieu, and Shraddha Shinde were employees and shareholders of Eli Lilly and Company during the time this work was conducted and analyzed.

Compliance with Ethics Guidelines. All procedures performed in studies involving human participants were in accordance with the ethical standards of the institutional and/or national research committee and with the 1964 Helsinki declaration and its later amendments or comparable ethical standards. The study protocol and all study procedures were approved by an institutional review board prior to initiating the study (Ethical and Independent Review Services, protocol \#20010-01, v1.0 date 06Jan2020, approved 11Feb2020). Informed consent was obtained from all individual participants included in the study.

Data Availability. Data are not publicly available but may be provided upon request.

Open Access. This article is licensed under a Creative Commons Attribution-NonCommercial 4.0 International License, which permits any non-commercial use, sharing, adaptation, distribution and reproduction in any medium 
or format, as long as you give appropriate credit to the original author(s) and the source, provide a link to the Creative Commons licence, and indicate if changes were made. The images or other third party material in this article are included in the article's Creative Commons licence, unless indicated otherwise in a credit line to the material. If material is not included in the article's Creative Commons licence and your intended use is not permitted by statutory regulation or exceeds the permitted use, you will need to obtain permission directly from the copyright holder. To view a copy of this licence, visit http://creativecommons.org/licenses/bync/4.0/.

\section{REFERENCES}

1. Hossain P, Kawar B, El Nahas M. Obesity and diabetes in the developing world: A growing challenge. N Engl J Med. 2007;356(3):213-5.

2. Wild S, Roglic G, Green A, Sicree R, King H. Global prevalence of diabetes: estimates for the year 2000 and projections for 2030. Diab Care. 2004;27(5): 1047-53.

3. World Health Organization (WHO). Diabetes: Global report on diabetes Geneva, Switzerland; 2016 [October 13, 2020]. 88 pgs. Available from: http:// apps.who.int/iris/bitstream/10665/204871/1/ 9789241565257_eng.pdf?ua=1.

4. Saeedi P, Petersohn I, Salpea P, Malanda B, Karuranga $S$, Unwin $N$, et al. Global and regional diabetes prevalence estimates for 2019 and projections for 2030 and 2045: Results from the International Diabetes Federation Diabetes Atlas, 9(th) edition. Diabetes Res Clin Pract. 2019;157:107843.

5. Bhupathiraju SN, Hu FB. Epidemiology of obesity and diabetes and their cardiovascular complications. Circ Res. 2016;118(11):1723-35

6. Chatterjee S, Khunti K, Davies MJ. Type 2 diabetes. Lancet. 2017;389(10085):2239-51.

7. Hu FB. Globalization of diabetes: the role of diet, lifestyle, and genes. Diab Care. 2011;34(6):1249-57.

8. Jaacks LM, Vandevijvere S, Pan A, McGowan CJ, Wallace $\mathrm{C}$, Imamura $\mathrm{F}$, et al. The obesity transition: stages of the global epidemic. Lancet Diabetes Endocrinol. 2019;7(3):231-40.
9. Kalyani RR, Golden SH, Cefalu WT. Diabetes and aging: unique considerations and goals of care. Diab Care. 2017;40(4):440-3.

10. Tabish SA. Is diabetes becoming the biggest epidemic of the twenty-first century? Int J Health Sci (Qassim). 2007;1(2):V-VIII.

11. Goldstein BJ. Insulin resistance: from benign to type 2 diabetes mellitus. Rev Cardiovasc Med. 2003;4(Suppl 6):S3-10.

12. Ogunmodede AJ, Abiodun $\mathrm{O}$, Makanjuola $\mathrm{AB}$, Olarinoye JK, Ogunmodede JA, Buhari OI. Burden of care and psychological distress in primary caregivers of patients with type -2 diabetes mellitus in A tertiary hospital in Nigeria. Ethiop J Health Sci. 2019;29(6):697-708.

13. Rubin RR, Peyrot M. Quality of life and diabetes. Diabetes Metab Res Rev. 1999;15(3):205-18.

14. Cannon A, Handelsman Y, Heile M, Shannon M. Burden of illness in type 2 diabetes mellitus. J Manag Care Spec Pharm. 2018;24(9-a Suppl): S5-S13.

15. Fowler MJ. Microvascular and macrovascular complications of diabetes. Clin Diabetes. 2008;26(2): 77-82.

16. Beverly EA, Ritholz MD, Brooks KM, Hultgren BA, Lee $Y$, Abrahamson MJ, et al. A qualitative study of perceived responsibility and self-blame in type 2 diabetes: reflections of physicians and patients. J Gen Intern Med. 2012;27(9):1180-7.

17. Cimo A, Dewa CS. Symptoms of mental illness and their impact on managing type 2 diabetes in adults. Can J Diabetes. 2018;42(4):372-81.

18. Peyrot M, Rubin RR, Lauritzen T, Snoek FJ, Matthews DR, Skovlund SE. Psychosocial problems and barriers to improved diabetes management: results of the Cross-National Diabetes Attitudes, Wishes and Needs (DAWN) Study. Diabet Med. 2005;22(10):1379-85.

19. American Diabetes Association (ADA). Standards of medical care in diabetes-2020 abridged for primary care providers. Clin Diabetes. 2020;38(1): 10-38.

20. Artasensi A, Pedretti A, Vistoli G, Fumagalli L. Type 2 diabetes mellitus: a review of multi-target drugs. Molecules. 2020;25(8).

21. Frias JP, Nauck MA, Van J, Kutner ME, Cui X, Benson C, et al. Efficacy and safety of LY3298176, a novel dual GIP and GLP-1 receptor agonist, in patients with type 2 diabetes: a randomised, 
placebo-controlled and active comparator-controlled phase 2 trial. Lancet. 2018;392(10160): 2180-93.

22. Friese S, Ringmayr T. ATLAS.ti 8 User Guide and Reference. Berlin: ATLAS.ti Scientific Software Development GmBH; 2020.

23. Hsieh HF, Shannon SE. Three approaches to qualitative content analysis. Qual Health Res. 2005;15(9):1277-88.

24. Willis GB. Analysis of the Cognitive Interview in Questionnaire Design. Understanding Qualitative Research. New York, NY: Oxford University Press; 2015.

25. Boeije H. A purposeful approach to the constant comparative method in the analysis of qualitative interviews. Qual Quant. 2002;36(4):391-409.

26. Litterbach E, Holmes-Truscott E, Pouwer F, Speight J, Hendrieckx C. 'I wish my health professionals understood that it's not just all about your HbA1c !'. Qualitative responses from the second Diabetes MILES - Australia (MILES-2) study. Diabetic Med. 2020;37(6):971-81.

27. Pal K, Dack C, Ross J, Michie S, May C, Stevenson F, et al. Digital health interventions for adults with type 2 diabetes: qualitative study of patient perspectives on diabetes self-management education and support. J Med Internet Res. 2018;20(2):e40.

28. St George SM, Pulgaron ER, Ferranti D, Agosto Y, Toro MI, Ramseur KC, et al. A qualitative study of cognitive, behavioral, and psychosocial challenges associated with pediatric type 2 diabetes in ethnic minority parents and adolescents. Diabetes Educ. 2017;43(2):180-9.

29. Tanenbaum ML, Kane NS, Kenowitz J, Gonzalez JS. Diabetes distress from the patient's perspective: Qualitative themes and treatment regimen differences among adults with type 2 diabetes. J Diabetes Complications. 2016;30(6):1060-8.

30. Wardian JL, Tate J, Folaron I, Graybill S, True M, Sauerwein T. Who's distressed? A comparison of diabetes-related distress by type of diabetes and medication. Patient Educ Couns. 2018;101(8): 1490-5.

31. Jeong M, Reifsnider E. Associations of diabetes-related distress and depressive symptoms with glycemic control in Korean Americans with type 2 diabetes. Diabetes Educ. 2018;44(6):531-40.

32. Mishra A, Podder V, Modgil S, Khosla R, Anand A, Nagarathna R, et al. Higher perceived stress and poor glycemic changes in prediabetics and diabetics among Indian population. J Med Life. 2020;13(2): 132-7.

33. Parsa S, Aghamohammadi M, Abazari M. Diabetes distress and its clinical determinants in patients with type II diabetes. Diabetes Metab Syndr. 2019;13(2):1275-9.

34. Ascher-Svanum H, Zagar A, Jiang D, Schuster D, Schmitt H, Dennehy EB, et al. Associations between glycemic control, depressed mood, clinical depression, and diabetes distress before and after insulin initiation: An exploratory. Post Hoc Anal Diabetes Ther. 2015;6(3):303-16.

35. Chen J, Fan L, Peng X, Ilag L, Ly T, Johnson J. Patient-reported outcomes in a study of human regular U-500 insulin delivered by continuous subcutaneous insulin infusion or multiple daily injections in patients with type 2 diabetes. Diabetes Obes Metab. 2020.

36. Zhu L, Chandran SR, Tan WB, Xin X, Goh SY, Gardner DSL. Persistent anxiety is associated with higher glycemia post-transition to adult services in Asian Young adults with diabetes. Diabetes Metab J. 2020. 